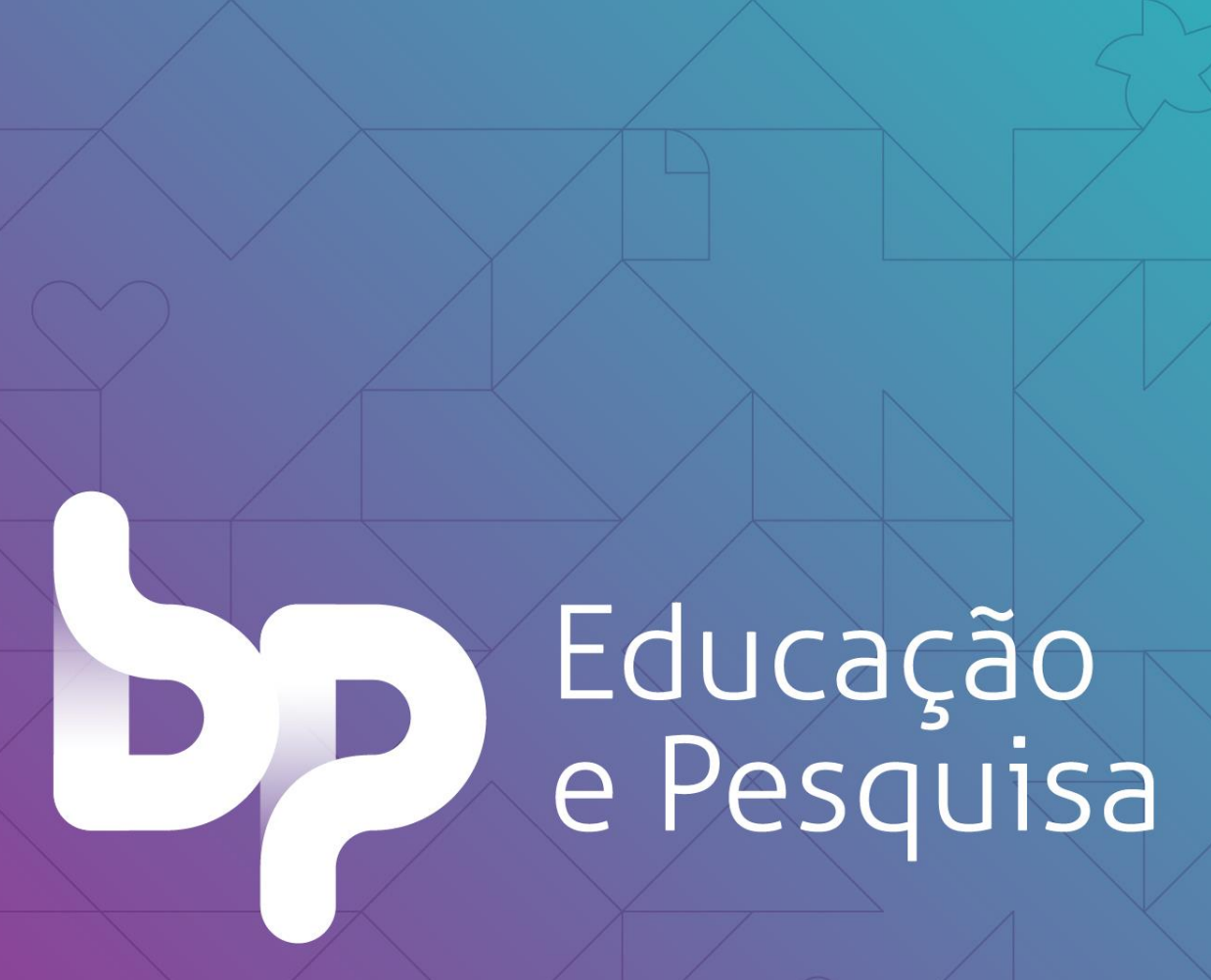




\section{Educação e Pesquisa}

RELATO DE EXPERIÊNCIA: PRÁTICA SIMULADA APLICADA PARA EQUIPE DE ENFERNAGEM NA IMPLANTACÂO DE UM NOVO SISTEMA DE TECNOLOGIA INFORMATIZADA EM HOSPITAL DE GRANDE PORTE.

Autora: Camilla do Rosário Nicolino Chiorino

Coautores: Kelle Cristina da Rocha,Ana Carolina Palmieri,Danielle Cristina Crespo,Silvia Helena Frota Mendonça 


\section{Apresentação da Instituição}

A Beneficência Portuguesa de São Paulo agora é BP, um polo de saúde composto por 7 unidades de negócio distintas, sendo 4 hospitais e 3 outros serviços de saúde e de educação e pesquisa. Fundada em 1859, a BP possui mais de 8.000 colaboradores e 4.500 médicos distribuídos em 8 edifícios e cerca de 50 clínicas nos bairros da Bela Vista, onde são concentrados os serviços privados, e da Penha, onde são

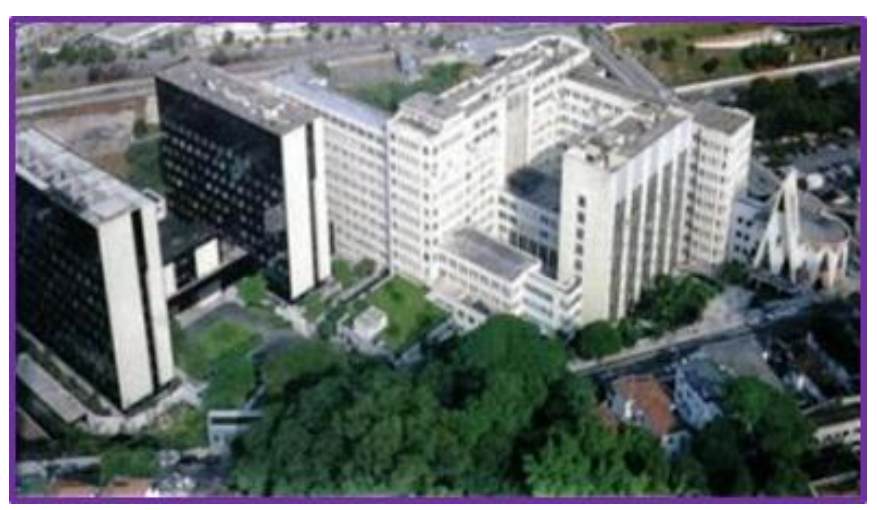
oferecidos os serviços para clientes encaminhados via regulação pelo Sistema Único de Saúde (SUS).

Hospital BP
R. Maestro Cardim. $n^{\circ} 769$

R. Maestro Cardim, nº 769

Tel. +55 (11) 3505-1000
BP Mirante

R. Martiniano de Carvalho, no 965

CEP: 01321-001 | Bela Vista | São Paulo - SP

Tel.: + 55 (11) 3505-1000
BP Hospital Filantrópico - Unidade Penha R. General Sócrates, $n^{\circ} 145$

S Tel. : +55 (11) 3505-1000

\section{bp \\ bp manac

bp

Medicina 


\section{Introdução}

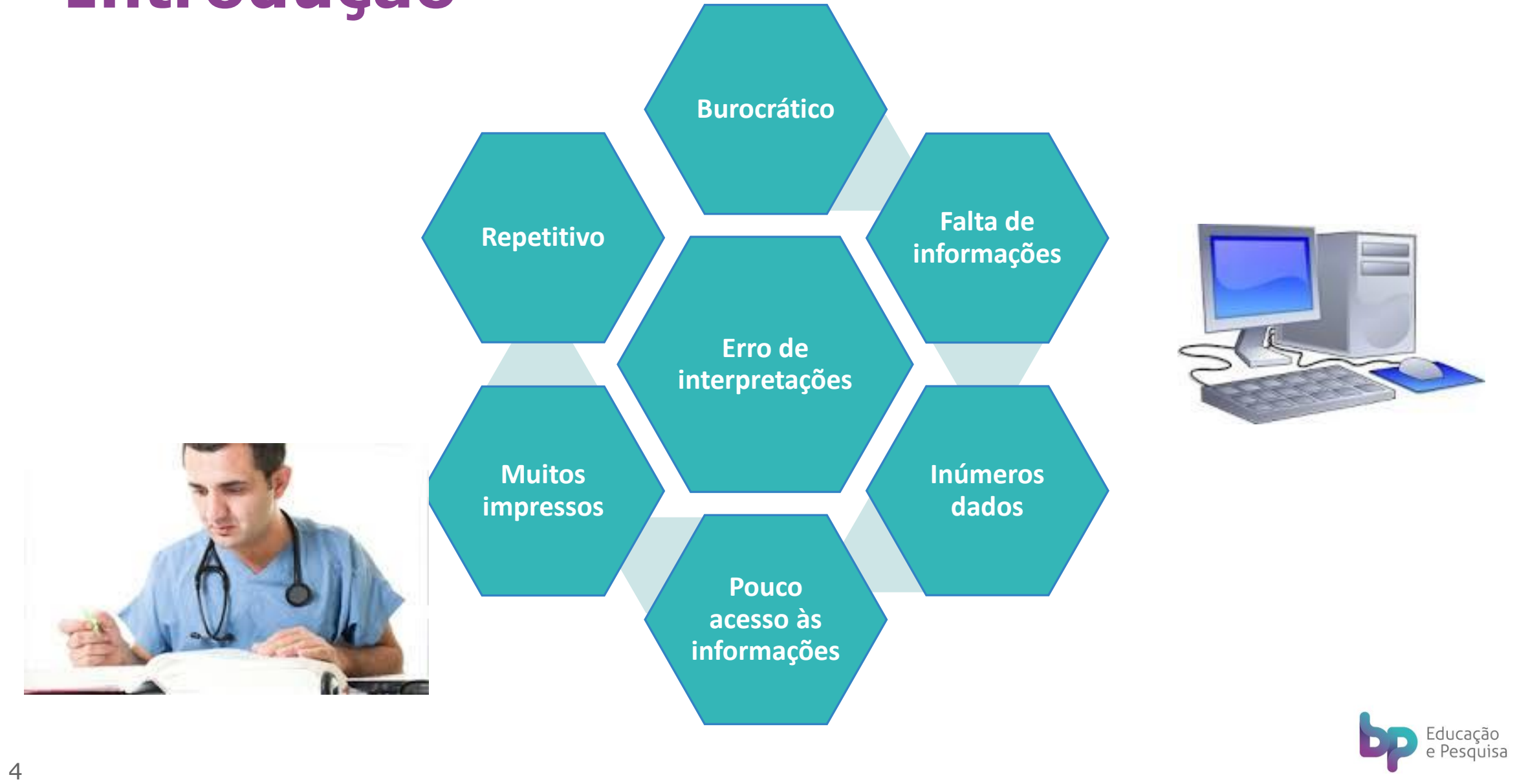




\section{De -> Para}

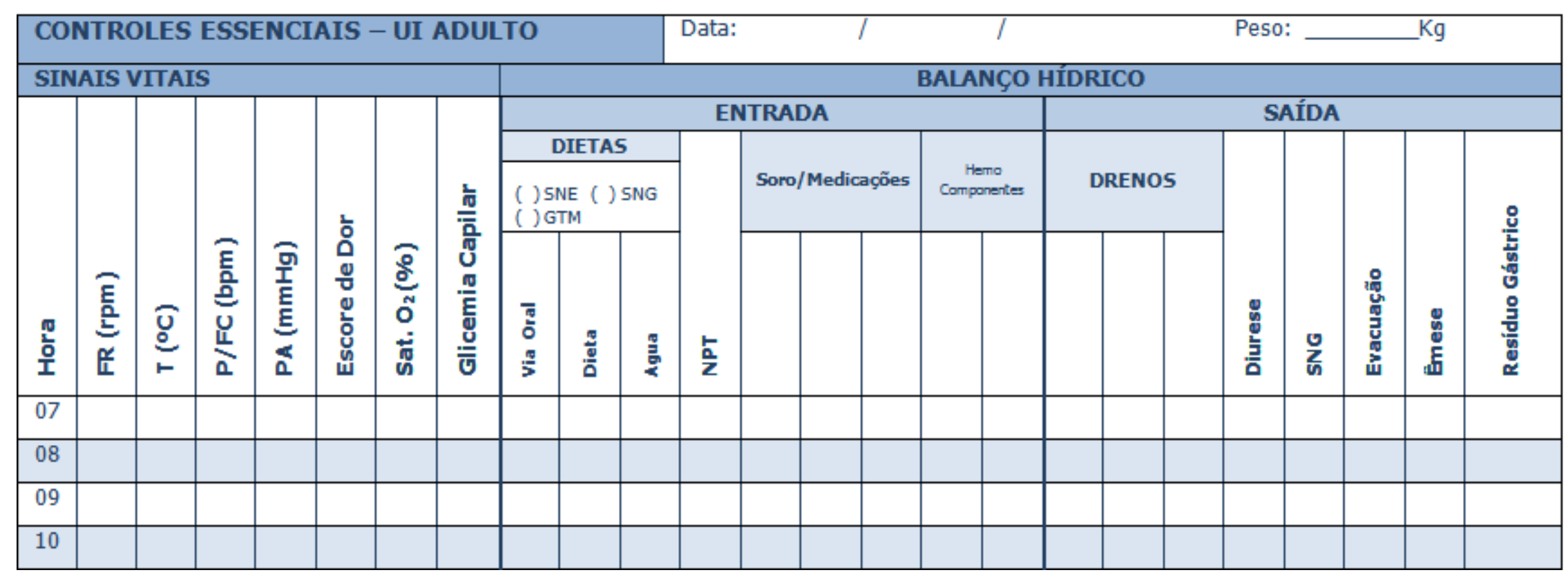




\section{Introdução}

A implantação de um novo sistema operacional informatizado em uma instituição de grande porte gera expectativas e ansiedades relacionadas à mudança de rotina que interfere diretamente nos cuidados assistenciais.

Para tanto, exige um treinamento que capacite e também permita ao usuário a experiência de transpor a mudança de sistema à sua realidade prática.

Frente a este cenário o recurso utilizado foi desenvolver o treinamento baseado na simulação prática para incorporação das rotinas assistenciais ao novo sistema informatizado. 


\section{Objetivo}

Descrever a prática simulada utilizada em treinamento, para a implantação de um sistema de tecnologia informatizada voltada à equipe de Enfermagem. 


\section{Relato de experiência}




\section{Plano de aula}

\section{PHILIPS}

\section{AA - Análise de Aderência}

\section{Enfermeiros UTI e UI}

\begin{tabular}{l|l}
\hline *Processo: Enfermagem UTI e UI & $\begin{array}{l}\text { *Responsável/Usuário-chave: Cristina } \\
\text { Satoko. Mizoi }\end{array}$ \\
\hline
\end{tabular}

\section{*Descrição Sucinta}

Possui profissionais de enfermagem qualificados em seus diferentes níveis e especializações, estando estes distribuídos nos diversos setores conforme dimensionamento instituído.

A SAE (Sistematização da Assistência de Enfermagem) é uma atividade privativa do enfermeiro, organizada em fases que estão interligadas e, é de fundamental importância que estas se articulem:

- Historico: Coleta de dados - anamnese e exame físico

- Diagnósticos / Problemas colaborativos

- Prescrição: Planejamento e Implementação

- Avaliação: Evolução

A instituição também contempla outros processos, que norteiam e evidenciam a assistência, os quais serão referenciados no documento.

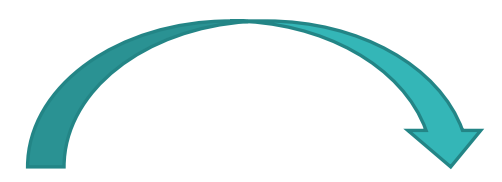

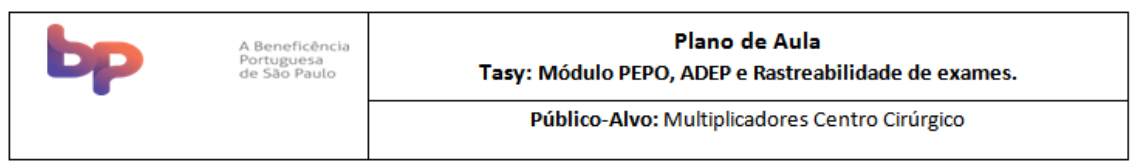

\begin{tabular}{|c|c|c|}
\hline CONTEÚDO PROGRAMÁTICO & ATIVIDADE DE ENSINO APRENDIZAGEM & $\begin{array}{l}\text { CARGA HORÁRIA } \\
\text { (8 horas) }\end{array}$ \\
\hline 1. Acessa o Tasy. & $\begin{array}{l}\text { Acessar o Tasy na base de treinamento com Usuário e } \\
\text { Senha determinados para o periodo de treinamento. }\end{array}$ & 5 minutos \\
\hline \multicolumn{3}{|c|}{ 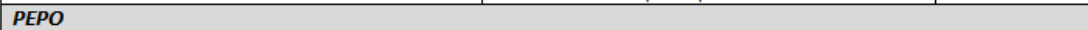 } \\
\hline 2. Localiza o Paciente. & $\begin{array}{l}\text { Localizar o paciente fictício na base de treinamento } \\
\text { através da agenda cirưrgica, com supervisấ e } \\
\text { acompanhamento do Instrutor facilitador. }\end{array}$ & 5 minutos \\
\hline $\begin{array}{l}\text { 3. Consulta à Anamnese, Diagnósticos, Cirurgia, } \\
\text { Cirurgia - resumo e descrições, Exames } \\
\text { laboratoriais e não laboratoriais, atestados, } \\
\text { medicamentos e parecer médico. }\end{array}$ & $\begin{array}{l}\text { Exemplificar consulta de Diagnósticos e Cirurgias. } \\
\text { Resumos e descriç̃es, exames laboratoriais e não } \\
\text { laboratoriais e parecer médico. }\end{array}$ & 5 minutos \\
\hline 4. Registra precauções & $\begin{array}{l}\text { Apresentar } 0 \text { registro de inserção ou retirada de } \\
\text { isolamento. }\end{array}$ & 10 minutos \\
\hline 5. Registra Escalas e Índices & Realizar os registros das escalas Elpg, Aldrets e Steward. & 15 minutos \\
\hline $\begin{array}{l}\text { 6. Realiza registro de Sinais Vitais e registro da dor } \\
\text { (salva, libera e inativa). }\end{array}$ & $\begin{array}{l}\text { Realizar registros de SSVV, e registros de avaliação da dor } \\
\text { conforme escalas padronizadas na instituição, através de } \\
\text { casos clínicos, com supervisão e acompanhamento do } \\
\text { Instrutor facilitador. }\end{array}$ & 10 minutos \\
\hline 7. Realiza anotação/evolução de enfermagem. & $\begin{array}{l}\text { Realizar anotação/evoluçăo de enfermagem no sistema } \\
\text { através de casos clínicos, com supervisão e } \\
\text { acompanhamento do Instrutor facilitador. }\end{array}$ & 10 minutos \\
\hline 8. Registra Perdas e ganhos & $\begin{array}{l}\text { Evidenciar a escolha do tipo de item (ganho ou perda), } \\
\text { evidenciar ganhos e perdas relacionados à irrigaçăo } \\
\text { vesical. }\end{array}$ & 15 minutos \\
\hline
\end{tabular}

https://proceedings.science/p/112281 ?lang=pt-br 


\section{Validação técnica}

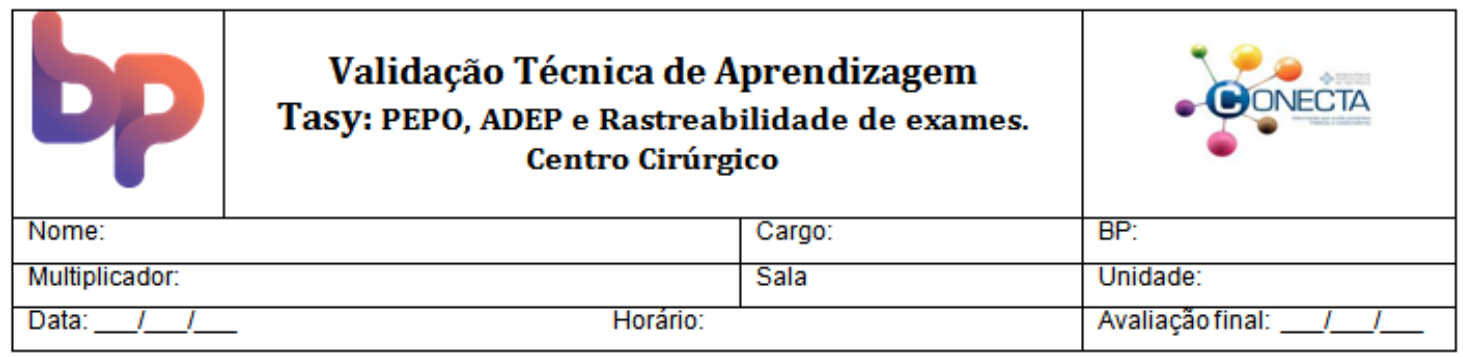

\begin{tabular}{|c|c|c|c|c|c|}
\hline \multirow[b]{2}{*}{ FUNÇÕES } & \multicolumn{2}{|c|}{ AUTOAVALIAÇĀO } & \multicolumn{2}{|c|}{ AVALIAÇĀO } & \multirow[b]{2}{*}{ Observações } \\
\hline & Satisfatório & Insatisfatónio & Satisfatório & Insatisfatório & \\
\hline 1. Acessa o Tasy. & & & & & \\
\hline \multicolumn{6}{|l|}{$>P E P O$} \\
\hline 2. Localiza o Paciente. & & & & & \\
\hline $\begin{array}{l}\text { 3. Consulta à Anamnese, Diagnósticos, Cirurgia, } \\
\text { Cirurgia - resumo e descrições, Exames } \\
\text { laboratoriais e não laboratoriais, parecer } \\
\text { médico, atestados, medicamentos. }\end{array}$ & & & & & \\
\hline 4. $\quad$ Registrar precauções & & & & & \\
\hline 5. Registra Escalas e índices & & & & & \\
\hline $\begin{array}{l}\text { 6. Realiza registro de Sinais Vitais e registro da } \\
\text { dor (salva, libera e inativa). }\end{array}$ & & & & & \\
\hline 7. Realiza anotação/evolução de enfermagem. & & & & & \\
\hline 8. $\quad$ Registra Perdas e ganhos & & & & & \\
\hline $\begin{array}{l}\text { 9. Realiza a inserção, retirada e troca de } \\
\text { dispositivos no prontuário. }\end{array}$ & & & & & \\
\hline
\end{tabular}

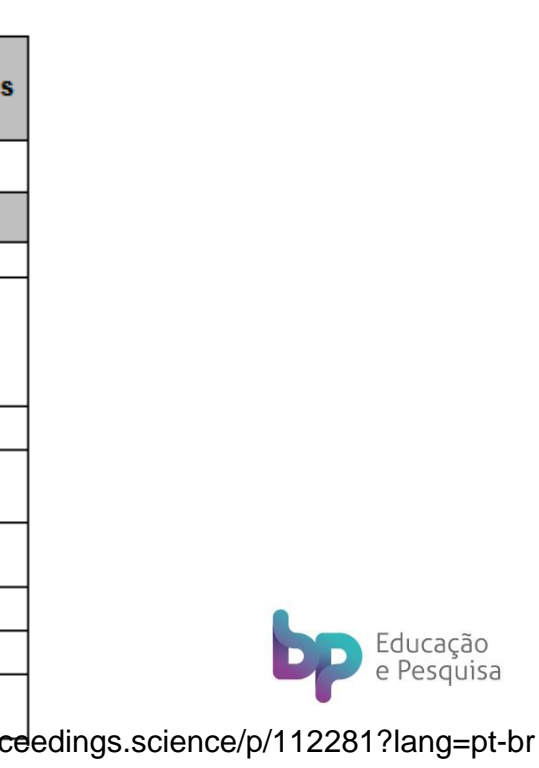




\section{Relato de experiência}
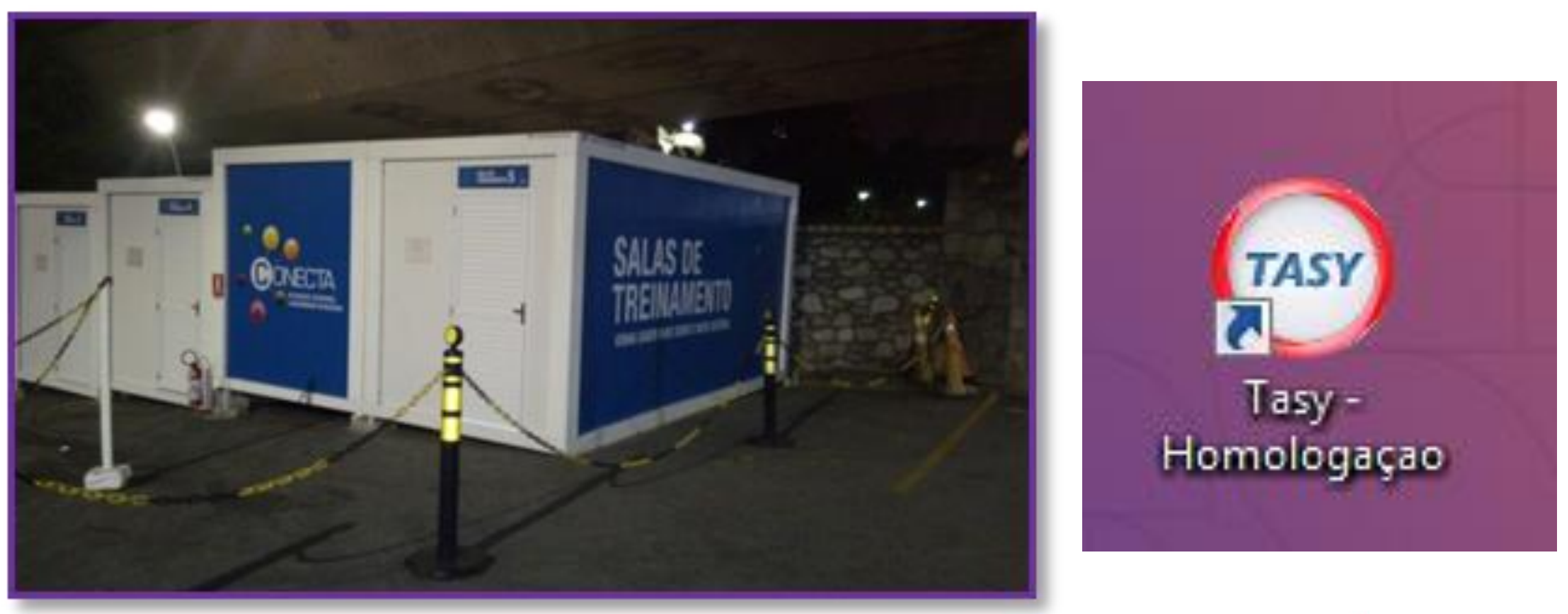


\section{Relato de experiência}
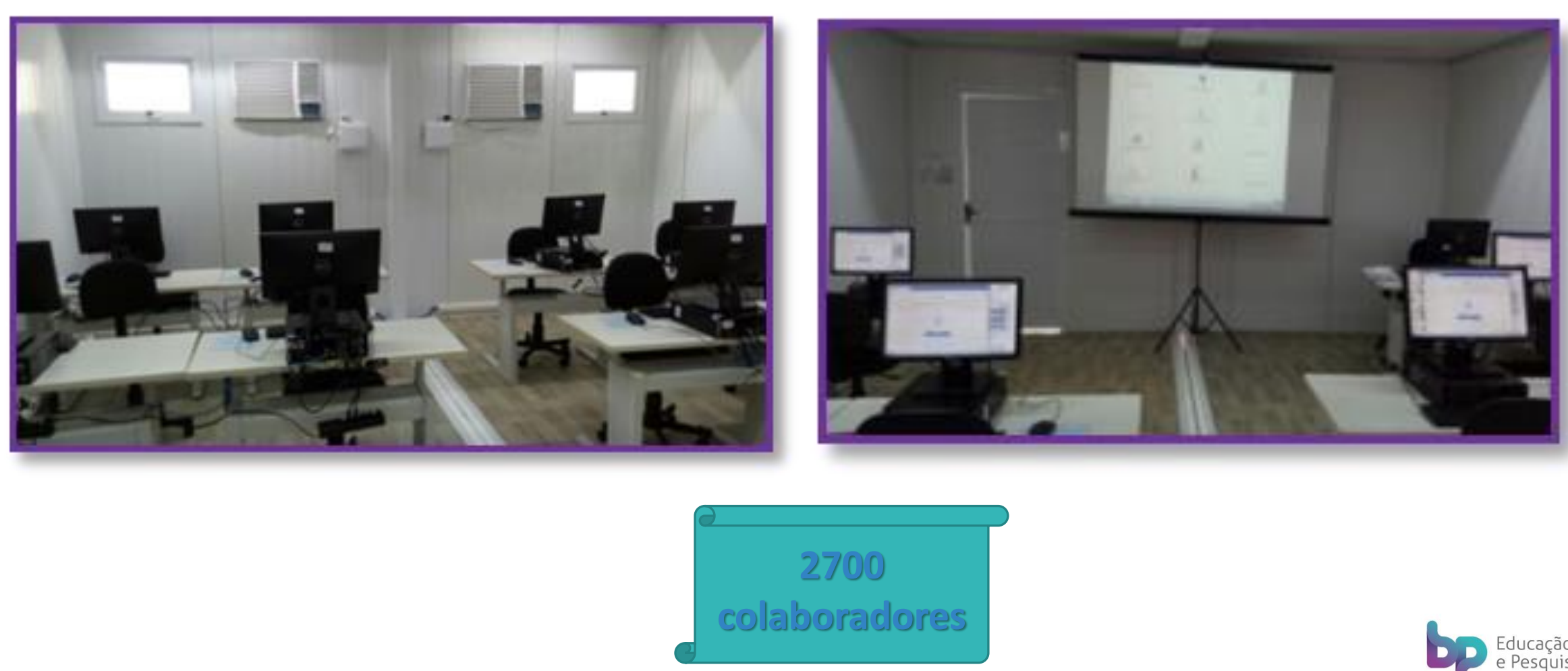

DD Educação 


\section{Resultados}




\section{Resultados}

A simulação prática em treinamento ofereceu a oportunidade de interagir com o novo sistema informatizado, diminuindo as expectativas e ansiedades. Permitiu, também, identificar fragilidades individuais para promover maior potencial de aprendizado e treinamento. 


\section{Considerações Finais}

A simulação prática, na medida em que refletiu aspectos reais da rotina assistencial dentro de um novo sistema operacional informatizado possibilitou a melhoria da base real do sistema, impactando diretamente na remodelação e produção de cadastros que antecediam o produto final deste novo sistema. 


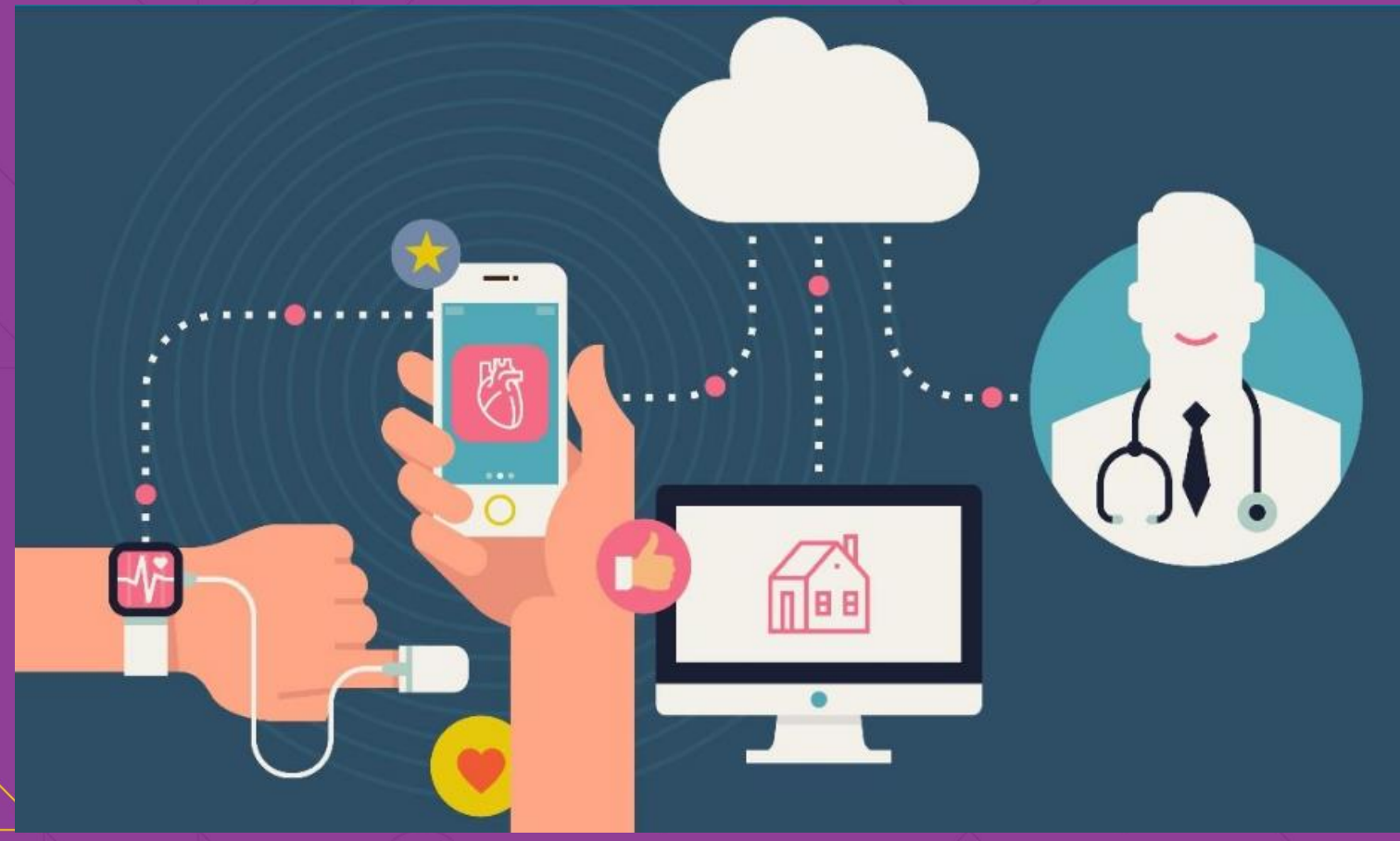




\section{Obrigada!}

\section{Camilla.chiorino@bp.org.br}

(11)3505-2015 